\title{
Three-step image rectification
}

\author{
Pascal MONASSE ${ }^{1}$ \\ monasse@imagine.enpc.fr \\ Jean-Michel MOREL ${ }^{2}$ \\ morel@cmla.ens-cachan.fr \\ Zhongwei TANG ${ }^{2}$ \\ tang@cmla.ens-cachan.fr
}

\author{
${ }^{1}$ IMAGINE, LIGM/Université \\ Paris-Est/École des Ponts ParisTech \\ Marne-la-Vallée, France \\ ${ }^{2}$ CMLA, ENS-Cachan \\ Cachan, France
}

\begin{abstract}
Image stereo-rectification is the process by which two images of the same solid scene undergo homographic transforms, so that their corresponding epipolar lines coincide and become parallel to the $x$-axis of image. A pair of stereo-rectified images is helpful for dense stereo matching algorithms. It restricts the search domain for each match to a line parallel to the $x$-axis. Due to the redundant degrees of freedom, the solution to stereorectification is not unique and actually can lead to undesirable distortions or be stuck in a local minimum of the distortion function. In this paper a robust geometric stereorectification method by a three-step camera rotation is proposed and mathematically explained. Unlike other methods which reduce the distortion by explicitly minimizing an empirical measure, the intuitive geometric camera rotation angle is minimized at each step. For un-calibrated cameras, this method uses an efficient minimization algorithm by optimizing only one natural parameter, the focal length. This is in contrast with all former methods which optimize between 3 and 6 parameters. Comparative experiments show that the algorithm has an accuracy comparable to the state-of-art, but finds the right minimum in cases where other methods fail, namely when the epipolar lines are far from horizontal.
\end{abstract}

\section{Introduction}

The stereo rectification of an image pair is an important component in many computer vision applications. The precise 3D reconstruction task requires an accurate dense disparity map, which is obtained by image registration algorithms. By estimating the epipolar geometry between two images and performing stereo-rectification, the search domain for registration algorithms is reduced and the comparison simplified, because horizontal lines with the same $y$ component in both images are in one to one correspondence. Stereo-rectification methods simulate rotations of the cameras to generate two coplanar image planes that are in addition parallel to the baseline.

From the algebraic viewpoint, the rectification is achieved by applying $2 \mathrm{D}$ projective transformations (or homographies) on both images. This pair of homographies is not unique, because a pair of stereo-rectified images remains stereo-rectified under a common rotation of both cameras around the baseline. This remaining degree of freedom can introduce an 
undesirable distortion to the rectified images. In the literature, several methods have been proposed to reduce this distortion. In $[\boldsymbol{\Xi}]$, authors first rectify one image and find another "matched" homography to rectify the other image. The distortion is reduced by imposing that one homography is approximately rigid around one point and by minimizing the $x$ disparity between both rectified images. In [四], the distortion reduction is improved by decomposing the homographies into three components: homograhy, similarity and shear. A projective transformation is sought, as affine as possible to reduce projective distortion, but the affine distortion is not treated. In [ $⿴ 囗 十$, a new parametrization of the fundamental matrix based on two rectification homographies is used to fit the feature correspondences. The rectification problem is formulated as a 6-parameter non-linear minimization problem. This method is very compact but no special attention is paid to the distortion reduction. In [0], the distortion is interpreted as local loss or creation of pixels in the rectified images. Thus the local area change in the rectified images is minimized. A similar idea is exposed in [D], whose solution is a homography that can be locally well approximated by a rigid transformation through the whole image domain. The rectification problem is also studied in the special situation where the camera projection matrix is known without explicitly reducing distortion [ $[$, 日]. Although different measures for rectification distortion are proposed in the above methods, the distortion is minimized explicitly. However it is not clear which measure would be more appropriate for image rectification. In the method proposed here, the distortion is not minimized explicitly. The rectification process is decomposed into three steps. The first step sends both epipoles to infinity; then the epipoles are sent to infinity in the $x$-direction; eventually the residual rotation between both cameras around their baseline is compensated to achieve the rectification. At each step, the camera rotation induces a homography on each image whose rotation angle is minimized to reduce the distortion. In contrast with the one-step rotation proposed in [⿴囗⿴囗十), we shall see that the three-step rotation makes the algorithm more robust. Even in extreme cases where the initial epipolar lines are far from horizontal, the algorithm works well while all other algorithms fail. The method yields a result, no matter whether the camera calibration matrix is known or not. In the latter case the proposed method can be easily formulated as a one-parameter minimization problem under the assumption of square aspect-ratio, zero skewness and image center as

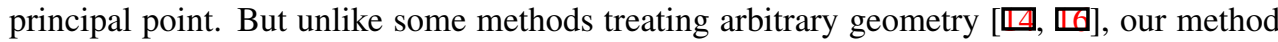
can only treat the case where the epipoles are outside the image domain. The method is detailed in Section 2. Some results are compared and commented in Section 3 followed by a conclusion in Section 4.

\section{Description of the method}

Space or image points will be denoted by lowercase bold letters and matrices by uppercase bold letters. The rectification works in the two-dimensional projective space $\mathscr{P}^{2}$. A point is a 3 -vector in $\mathscr{P}^{2}$, for example, $\mathbf{m}=(x, y, w)^{T}$, corresponding to the Euclidean point $(x / w, y / w)^{T}$. If $w=0$, then the point is at infinity in the $(x, y)$ direction. A transformation in the two-dimensional projective space $\mathscr{P}^{2}$ is a $3 \times 3$ matrix. Examples of such transformations are the fundamental matrix, denoted by $\mathbf{F}$ and homographies denoted by $\mathbf{H}$.

As usual in stereo-rectification, a set of non-degenerate correspondences between image $I_{1}$ and $I_{2}$ are given, permitting to compute the correct fundamental matrix $\mathbf{F}$. For that purpose,

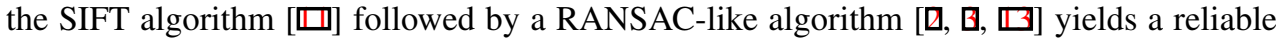
enough input with inliers only. 


\subsection{Rectification geometry}

The fundamental matrix corresponds to two stereo-rectified images if and only if it has the special form (up to a scale factor)

$$
[\mathbf{i}]_{\times}=\left[\begin{array}{c}
1 \\
0 \\
0
\end{array}\right]_{\times}=\left[\begin{array}{ccc}
0 & 0 & 0 \\
0 & 0 & -1 \\
0 & 1 & 0
\end{array}\right] .
$$

Having both cameras pointing to the same direction with their image planes co-planar and parallel to the baseline is still not sufficient to achieve rectification. Assume the cameras to have the form $\mathbf{P}=\mathbf{K}[\mathbf{I} \mid \mathbf{0}]$ and $\mathbf{P}^{\prime}=\mathbf{K}^{\prime}[\mathbf{I} \mid \mathbf{i}]$ with the motion between both cameras being only the translation along the $x$-axis. Then the fundamental matrix is proportional to $[\mathbf{i}]_{\times}$if and only if $\mathbf{K}$ and $\mathbf{K}^{\prime}$ have the same second row.

The orientation of the camera can be adjusted by applying a homography on the image, which has the form:

$$
\mathbf{H}=\mathbf{K R K}^{-1}
$$

where $\mathbf{R}$ is the relative rotation before and after rectification. Is the rectification achieved by finding a pair of homographies which sends the epipoles in each image to $(1,0,0)^{T}(x$ direction)? The answer is NO. Having the epipoles at $(1,0,0)^{T}$ only means the relationship between two cameras is a rotation around the baseline and images are generally unrectified.

\subsection{Three-step rectification}

Assume cameras are not calibrated but have the same simple calibration matrix $\mathbf{K}$ before and after rectification:

$$
\mathbf{K}=\left[\begin{array}{lll}
f & 0 & \frac{w}{2} \\
0 & f & \frac{h}{2} \\
0 & 0 & 1
\end{array}\right]
$$

with $w, h$ the width and height of the image and $f$ the unknown focal length. The fundamental matrix $\mathbf{F}$ is computed from a group of non-degenerate correspondences between two images. The epipoles for the left image $\mathbf{e}=\left(e_{x}, e_{y}, 1\right)^{T}$ and right image $\mathbf{e}^{\prime}=\left(e_{x}^{\prime}, e_{y}^{\prime}, 1\right)^{T}$ can be computed as right and left null vectors of $\mathbf{F}: \mathbf{F e}=0$ and $\mathbf{e}^{\prime T} \mathbf{F}=0$.

The idea is to transform both images so that the fundamental matrix gets the form $[\mathbf{i}]_{\times}$. Unlike the other methods which directly parameterize the homographies from the constraints $\mathbf{H e}=\mathbf{i}, \mathbf{H}^{\prime} \mathbf{e}^{\prime}=\mathbf{i}$ and $\mathbf{H}^{\prime T}[\mathbf{i}]_{\times} \mathbf{H}=\mathbf{F}$ and find an optimal pair by minimizing a measure of distortion, we shall compute the homography by explicitly rotating each camera around its optical center. The algorithm is decomposed into three steps (Fig. 1):

1. Compute homographies $\mathbf{H}_{1}$ and $\mathbf{H}_{1}^{\prime}$ by rotating both cameras respectively so that the left epipole $\left(e_{x}, e_{y}, 1\right)$ is transformed to $\left(e_{x}, e_{y}, 0\right)$ and the right epipole $\left(e_{x}^{\prime}, e_{y}^{\prime}, 1\right)$ to $\left(e_{x}^{\prime}, e_{y}^{\prime}, 0\right)$.

2. Rotate both cameras so that $\left(e_{x}, e_{y}, 0\right)$ is transformed to $(1,0,0)$ and $\left(e_{x}^{\prime}, e_{y}^{\prime}, 0\right)$ to $(1,0,0)$. The corresponding homographies are denoted by $\mathbf{H}_{2}$ and $\mathbf{H}_{2}^{\prime}$.

3. Rotate one camera or both cameras together to compensate the residual relative rotation between both cameras around the baseline. The corresponding homographies are denoted by $\mathbf{H}_{3}$ and $\mathbf{H}_{3}^{\prime}$. 


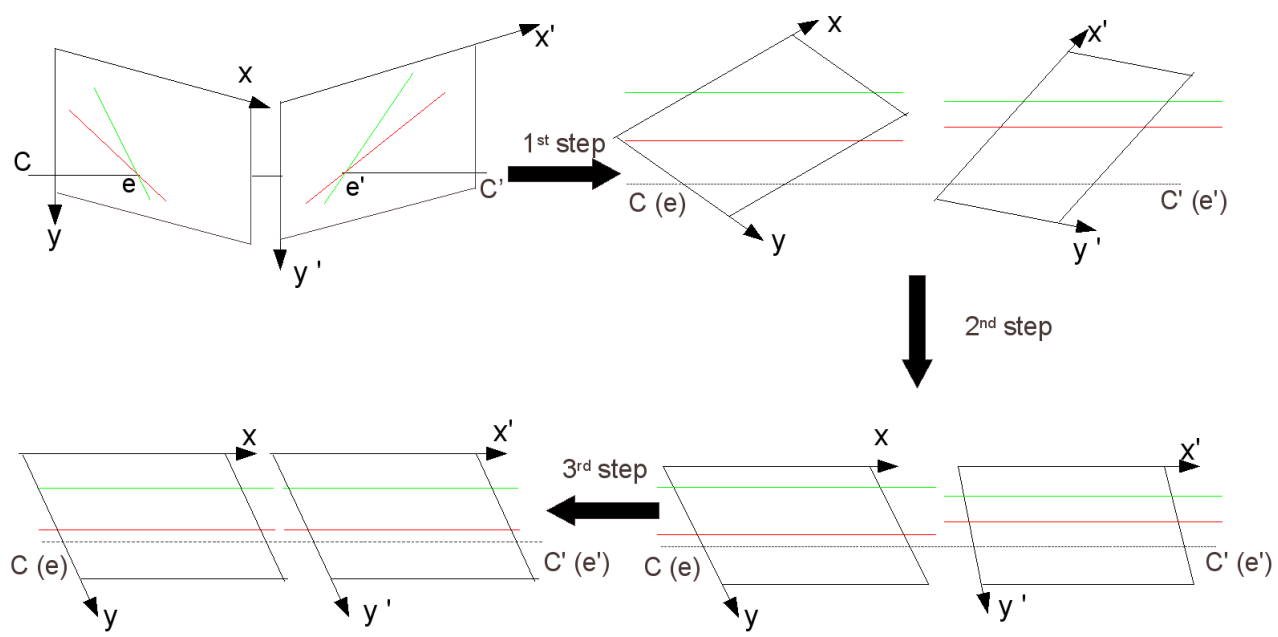

Figure 1: Three-step rectification. First step: the image planes become parallel to $C C^{\prime}$. Second step: the images rotate in their own plane to have their epipolar lines also parallel to $C C^{\prime}$. Third step: a rotation of one of the image planes around $C C^{\prime}$ aligns corresponding epipolar lines in both images. Note how the pairs of epipolar lines become aligned.

For the first step, we have the relationships: $\mathbf{H}_{1} \mathbf{e}=\left(e_{x}, e_{y}, 0\right)^{T}$ and $\mathbf{H}_{1}^{\prime} \mathbf{e}^{\prime}=\left(e_{x}^{\prime}, e_{y}^{\prime}, 0\right)^{T}$ with $\mathbf{H}_{1}=\mathbf{K} \mathbf{R} \mathbf{K}^{-1}$ and $\mathbf{H}_{1}^{\prime}=\mathbf{K R}^{\prime} \mathbf{K}^{-1}$. The solution for the rotation matrices is not unique. Thus we determine a rotation matrix with a minimal rotation angle, so that it introduces a minimal distortion. By rewriting $\mathbf{H}_{1} \mathbf{e}=\left(e_{x}, e_{y}, 0\right)^{T}$ as $\mathbf{R} \mathbf{K}^{-1} \mathbf{e}=\mathbf{K}^{-1}\left(e_{x}, e_{y}, 0\right)^{T}$, the problem is in fact to find a rotation matrix which rotates the vector $\mathbf{a}=\mathbf{K}^{-1} \mathbf{e}$ to $\mathbf{b}=\mathbf{K}^{-1}\left(e_{x}, e_{y}, 0\right)^{T}$. So the minimal angle $\theta$ is $\operatorname{acos}\left(\frac{\mathbf{a} \cdot \mathbf{b}}{|\mathbf{a}||\mathbf{b}|}\right)$ and the rotation axis $\mathbf{t}$ is $\frac{\mathbf{a} \times \mathbf{b}}{|\mathbf{a}||\mathbf{b}|}$. By Rodrigues formula, the rotation can be written as $\mathbf{R}(\theta, \mathbf{t})=\mathbf{I}+\sin \theta[\mathbf{t}]_{\times}+(1-\cos \theta)[\mathbf{t}]_{\times}^{2}$. This process can be repeated in step 2. After two steps, both epipoles are transformed to $(1,0,0)^{T}$. Notice that $\mathbf{H}_{1}, \mathbf{H}_{1}^{\prime}, \mathbf{H}_{2}$ and $\mathbf{H}_{2}^{\prime}$ are all parametrized by $f$.

The rectification is not finished and the fundamental matrix has only 0 in first row and first column. If the correct $\mathbf{K}$ is known and the lens distortion removed, it can be proven that the remaining relationship between two cameras is a rotation $\hat{\mathbf{R}}$ around the baseline.

Then the new fundamental matrix is $\hat{\mathbf{F}}=\mathbf{K}^{-T}[\mathbf{i}]_{\times} \hat{\mathbf{R}} \mathbf{K}^{-1}$ and the corresponding essential matrix $\hat{\mathbf{E}}=[\mathbf{i}]_{\times} \hat{\mathbf{R}}$. According to $[\mathbf{\theta}], \hat{\mathbf{R}}$ can be extracted from $\hat{\mathbf{E}}$, but an arbitrary $\mathbf{K}$ will not be compatible with $\hat{\mathbf{F}}$ and no rotaion can be extracted. By writing $\hat{\mathbf{E}}=\mathbf{U D V}^{\prime}$, one possible modification is to force the first two singular values of $\hat{\mathbf{E}}$ to be equal and the third one to 0 , which gives $\hat{\tilde{\mathbf{E}}}=\mathbf{U}\left[\begin{array}{lll}1 & 0 & 0 \\ 0 & 1 & 0 \\ 0 & 0 & 0\end{array}\right] \mathbf{V}^{T}$ and $\hat{\tilde{\mathbf{F}}}=\mathbf{K}^{-T} \hat{\tilde{\mathbf{E}}} \mathbf{K}^{-1}$. This modification is the smallest in the sense of Frobenius norm. Then the third rectification step can be pursued by using the extracted rotation matrix from $\hat{\tilde{\mathbf{E}}}$. But this modification can also change a lot the epipolar geometry. This can be evaluated by checking the sum of distances from the points to the modified epipolar lines,

$$
S(f)=\sum_{i=1}^{N} d\left(\mathbf{x}_{i}^{\prime}, \tilde{\mathbf{F}} \mathbf{x}_{i}\right)+d\left(\mathbf{x}_{i}, \tilde{\mathbf{F}}^{T} \mathbf{x}_{i}^{\prime}\right)
$$


with $\tilde{\mathbf{F}}=\mathbf{H}_{1}^{\prime T} \mathbf{H}_{2}^{\prime T} \hat{\tilde{\mathbf{F}}} \mathbf{H}_{2} \mathbf{H}_{1}$. An optimal $\mathbf{K}$ can be found by minimizing the distance function $S(f) . S(f)$ is a non-linear function of the focal length $f$. The Levenberg-Marquardt minimization algorithm $[\square]$ was chosen to find an optimal $f$. For more stability and efficiency, the Jacobian matrix $\frac{\partial S(f)}{\partial f}$ is computed explicitly instead of using a finite difference scheme. The delicate part of the Jacobian computation is $\frac{\partial \hat{\mathbf{E}}}{\partial f}$, which can be resolved by using a method proposed in [ $\square$ ]. Once the optimal $\mathbf{K}$ is found, $\mathbf{H}_{3}$ and $\mathbf{H}_{3}^{\prime}$ can be computed from the residual rotation.

It might be argued that one step of rotation is enough to rectify the images instead of three steps. But the three steps procedure makes the algorithm much more robust and, as we shall see, the residual distortion is equal or only slightly higher. In fact, the idea of parametrization of the fundamental matrix by just one step of camera rotation is used in [ 0 ]. As we will see in the experiments, this parametrization is not robust when the initial epipolar lines are far from horizontal.

\section{Results}

In this section, the algorithm is tested on several pairs of real images. In Table 1, the first three pairs of images are from Mallon's test set [D], which are taken by the same camera under a fixed lens configuration. Concerning the other three pairs of images, the camera motion causes the initial epipolar lines to be far from the horizontal direction. For all pairs, enough correct correspondences are available. The fundamental matrix was computed by the normalized 8-point algorithm [ $[\square]$ and the calibration matrix is unknown. The performance

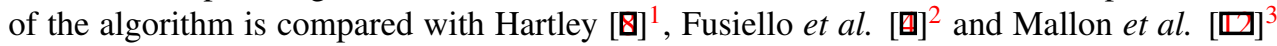
methods.

The performance is evaluated on two aspects: the rectification error and the introduced distortion. The rectification error is measured as the average and standard deviation of the $y$-disparity of rectified correspondences. The same statistics are computed for the original epipolar geometry with the distance from points to the corresponding epipolar lines as metric.

The distortion reduction is measured by two traditional criteria: orthogonality and aspect ratio. Consider the rectification homography $\mathbf{H}$ and four cross points $\mathbf{a}=\left(\frac{w}{2}, 0,1\right)^{T}, \mathbf{b}=$ $\left(w, \frac{h}{2}, 1\right)^{T}, \mathbf{c}=\left(\frac{w}{2}, h, 1\right)^{T}, \mathbf{d}=\left(0, \frac{h}{2}, 1\right)^{T}$. with $w$ and $h$ the width and the height of image. The orthogonality $\theta_{o}$ is defined as the angle between the vector $\mathbf{m}=\mathbf{H b}-\mathbf{H d}$ and $\mathbf{n}=\mathbf{H c}-\mathbf{H a}$ : $\theta_{o}=\cos ^{-1}\left(\frac{\mathbf{m} \cdot \mathbf{n}}{|\mathbf{m}||\mathbf{n}|}\right)$. The ideal value of orthogonality is $90^{\circ}$. By redefining $\mathbf{a}=(0,0,1)^{T}$, $\mathbf{b}=(w, 0,1)^{T}, \mathbf{c}=(w, h, 1)^{T}, \mathbf{d}=(0, h, 1)^{T}$, the aspect ratio $r_{d}$ is the length ratio between diagonals: $r_{d}=\left(\left(\mathbf{m}^{T} \mathbf{m}\right) /\left(\left(\mathbf{n}^{T} \mathbf{n}\right)\right)\right)^{1 / 2}$. The ideal value of the aspect ratio is 1 .

We argue that the above criteria are not sufficient. A rectification should also have a geometric meaning. For some pairs of images, we can deduce the rectified images since the camera motion is evident. This gives an empirical evaluation of the geometric meaning of the rectification.

The results are gathered in Table 1. In the first two examples ("Boxes" and "Arch", Fig. 2), the performance of the three algorithms are similar except that Hartley's method introduces more distortion. This is not surprising because the distortion is just reduced by

\footnotetext{
${ }^{1}$ Du Huynh's version: http: //www. csse.uwa.edu.au/ du/Software/rectification/

${ }^{2}$ Code available at: http://profs.sci.univr.it/ fusiello/demo/rect/.

${ }^{3}$ Only the first three example in Table 1 are tested by Mallon et al.'s since the code is not available.
} 
minimizing the disparity in $x$-axis, which is not directly related to distortion. In the third example ("Drive"), the proposed method has a slightly larger rectification error, but the rectification error is still coherent with the original error and in a reasonable range.

Fusiello et al.'s method is very competitive, in particular for the rectification precision. But its result does not always have a correct geometric meaning. In the example of "Building" (Fig. 3) and "Tower", two pairs of aerial images were taken by a camera installed on a helicopter. Since the motion is close to the $y$-axis of the camera, the initial epipolar lines are close to vertical. In such situation, a correct rectification algorithm should rotate both cameras so that the baseline is parallel to the $x$-axis. Only our algorithm rotates the images and therefore gives a small rectification error. Both Fusiello's method and Hartley's method fail, being stuck in a local minimum. In the example of "Cournot" (Fig. 4), the initial epipolar lines were also far from the horizontal direction. The images should have been rotated to achieve a good rectification. Even though Fusiello's method gives a result with a small rectification error, the geometry of the rectified images is not correct.

Notice that for Hartley's method the orthogonality of the homography for the right image is always $90^{\circ}$. Indeed the right homography has the form GRT where $\mathbf{R}$ is a rotation matrix and $\mathbf{T}$ a translation matrix. $\mathbf{G}$ is close to a rigid transformation if the epipole is far from the image domain, which is the case of the images in the experiments. The same phenomenon can be observed for the orthogonality for the left homography of Fusiello et al.'s method. In their algorithm, the left camera does not rotate around $x$-axis. And the rotation around $y$-axis and $z$-axis is also very small if the epipole is far away.

\section{Conclusion}

A new image rectification algorithm is proposed. This algorithm is decomposed into three steps of camera rotation. By computing the minimal rotation angle at each step, the distortion is implicitly limited. This algorithm performs as well as state-of-art algorithms, but for image pairs where the initial epipolar lines are far from horizontal, the fact that we have a unique parameter to estimate (focal length) makes the algorithm more robust by reducing the risks of reaching a local minimum.

\section{Acknowdledgments}

Part of this work was supported by the Agence Nationale de la Recherche (ANR), Callisto project (ANR-09-CORD-003).

\section{References}

[1] N. Ayache and C. Hansen. Rectification of images for binocular and trinocular stereovision. ICPR, 1988.

[2] M.A. Fischler and R.C. Bolles. Random sample consensus: A paradigm for model fitting with applications to image analysis and automated cartography. CACM, 24(3):381-395, 1981.

[3] Jan-Michael Frahm and Marc Pollefeys. Ransac for (quasi-)degenerate data (qdegsac). CVPR, 1, 2006.

[4] A. Fusiello and L. Irsara. Quasi-Euclidean uncalibrated epipolar rectification. ICPR, 2008.

[5] A. Fusiello, E. Trucco, and A. Verri. A compact algorithm for rectification of stereo pairs. Machine Vision and Applications, 12(1):16-22, 2000. 


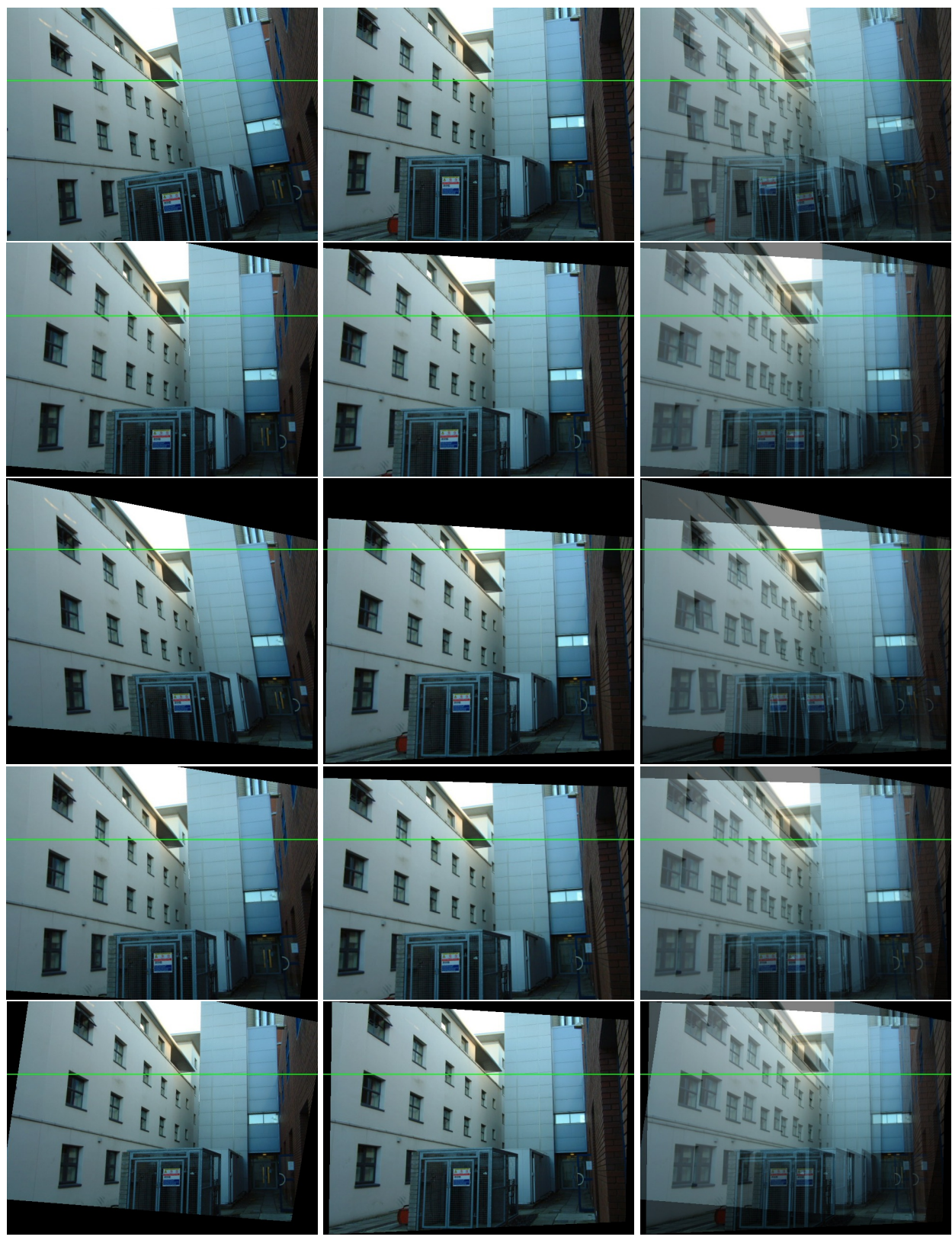

Figure 2: Image pair "Arch" rectified by different methods. From top to bottom: original images, proposed method, Hartley method, Fusiello et al. method and Mallon et al. method. A horizontal line is added to images to check the rectification. The third column represents an image average of each pair. 


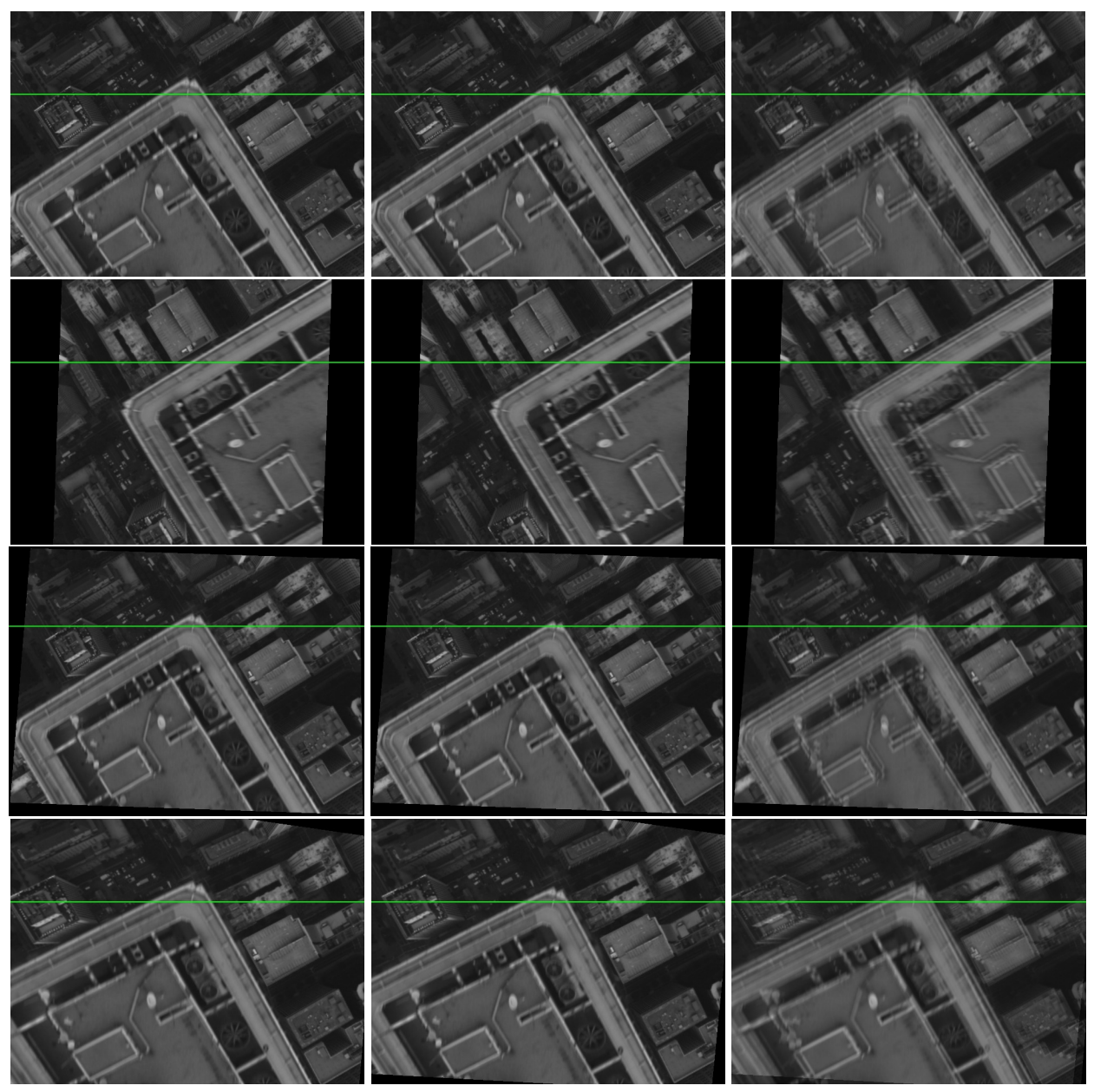

Figure 3: Image pair "Building" rectified by different methods. From top to bottom: original images, proposed method, Hartley method and Fusiello et al. method. A horizontal line is added to images to check the rectification. The third column represents an image average of each pair. 


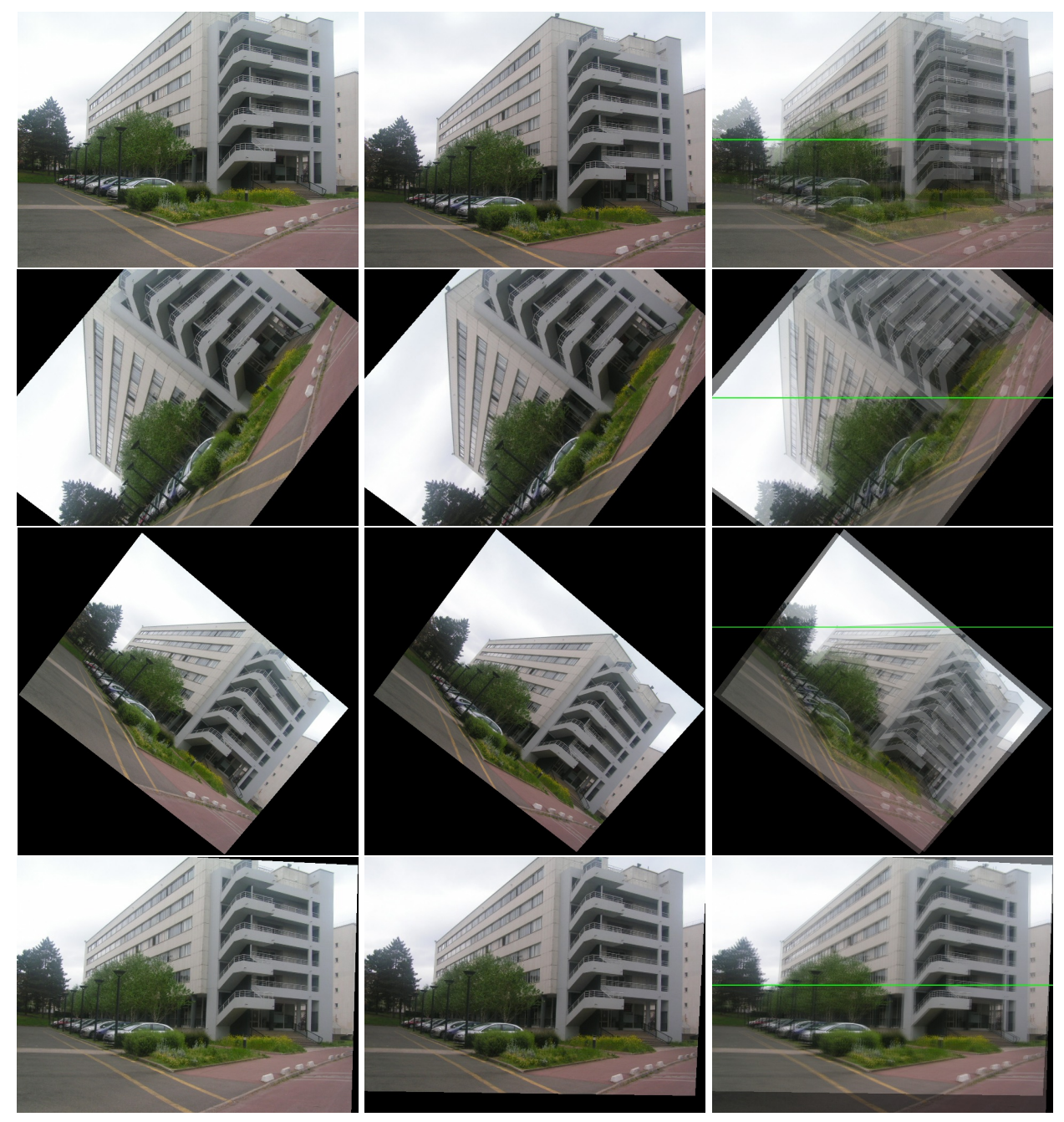

Figure 4: Image pair "Cournot" rectified by different methods. From top to bottom: original images, proposed method, Hartley method and Fusiello et al. method. A horizontal line is added to images to check the rectification. The third column represents an image average of each pair. 
Table 1: The performance comparison between the proposed method, Hartley's method [ब], Fusiello et al.'s method [四] and Mallon et al.'s method []]. The comparison is based on rectification error, orthogonality and aspect ratio. The ideal value of orthogonality and aspect ratio are $90^{\circ}$ and 1 respectively.

\begin{tabular}{|c|c|c|c|c|c|c|c|c|}
\hline \multirow{2}{*}{ Sample } & \multirow{2}{*}{ F Mat. } & \multirow{2}{*}{ Method } & \multicolumn{2}{|c|}{ Orthogonality } & \multicolumn{2}{|c|}{ Aspect ratio } & \multicolumn{2}{|c|}{ Rectification } \\
\hline & & & H & $\mathbf{H}^{\prime}$ & $\mathbf{H}$ & $\mathbf{H}^{\prime}$ & mean & std \\
\hline \multirow{4}{*}{ Boxes } & \multirow{4}{*}{$\begin{array}{l}0.1213 \\
0.0963\end{array}$} & Proposed & 89.60 & 89.63 & 0.9884 & 0.9892 & 0.1293 & 0.0887 \\
\hline & & Hartley & 94.07 & 90.00 & 1.0639 & 0.9948 & 0.1194 & 0.0968 \\
\hline & & Fusiello & 90.00 & 90.16 & 1.0000 & 1.0043 & 0.1055 & 0.0891 \\
\hline & & Mallon & 89.33 & 88.78 & 0.9889 & 0.9878 & 0.44 & 0.33 \\
\hline \multirow{4}{*}{ Arch } & \multirow{4}{*}{$\begin{array}{l}0.2107 \\
0.2247\end{array}$} & Proposed & 89.80 & 90.05 & 0.9942 & 1.0014 & 0.2520 & 0.2349 \\
\hline & & Hartley & 82.80 & 90.00 & 0.8841 & 1.0002 & 0.2089 & 0.2199 \\
\hline & & Fusiello & 90.00 & 90.19 & 1.0000 & 1.0051 & 0.2134 & 0.2593 \\
\hline & & Mallon & 90.26 & 91.22 & 1.0045 & 1.0175 & 0.22 & 0.33 \\
\hline \multirow{4}{*}{ Drive } & \multirow{4}{*}{$\begin{array}{l}0.5111 \\
0.7445\end{array}$} & Proposed & 89.95 & 90.00 & 0.9977 & 1.0001 & 0.7139 & 0.8253 \\
\hline & & Hartley & 91.96 & 90.00 & 1.0320 & 1.0001 & 0.5132 & 0.7462 \\
\hline & & Fusiello & 90.00 & 90.11 & 1.0000 & 1.0026 & 0.4962 & 0.7851 \\
\hline & & Mallon & 90.12 & 90.44 & 1.0021 & 1.0060 & 0.18 & 0.91 \\
\hline \multirow{4}{*}{ Building } & \multirow{4}{*}{$\begin{array}{l}0.1308 \\
0.1221\end{array}$} & Proposed & 89.96 & 89.95 & 0.9990 & 0.9989 & 0.1330 & 0.1242 \\
\hline & & Hartley & 90.02 & 90.00 & 1.0002 & 0.9998 & 6.4910 & 5.4584 \\
\hline & & Fusiello & 90.00 & 89.85 & 1.0000 & 0.9970 & 3.0508 & 2.4809 \\
\hline & & Mallon & $\mathrm{n} / \mathrm{a}$ & $\mathrm{n} / \mathrm{a}$ & $\mathrm{n} / \mathrm{a}$ & $\mathrm{n} / \mathrm{a}$ & $\mathrm{n} / \mathrm{a}$ & $\mathrm{n} / \mathrm{a}$ \\
\hline \multirow{4}{*}{ Tower } & \multirow{4}{*}{$\begin{array}{l}0.1370 \\
0.1154\end{array}$} & Proposed & 89.99 & 89.98 & 0.9998 & 0.9995 & 0.1438 & 0.1192 \\
\hline & & Hartley & 89.94 & 90.00 & 0.9990 & 0.9999 & 11.1079 & 3.5541 \\
\hline & & Fusiello & 90.00 & 89.89 & 1.0000 & 0.9979 & 3.2698 & 1.7266 \\
\hline & & Mallon & $\mathrm{n} / \mathrm{a}$ & $\mathrm{n} / \mathrm{a}$ & $\mathrm{n} / \mathrm{a}$ & $\mathrm{n} / \mathrm{a}$ & $\mathrm{n} / \mathrm{a}$ & $\mathrm{n} / \mathrm{a}$ \\
\hline \multirow{4}{*}{ Cournot } & \multirow{4}{*}{$\begin{array}{l}0.2055 \\
0.1563\end{array}$} & Proposed & 89.66 & 89.29 & 0.9920 & 0.9833 & 0.3242 & 0.2082 \\
\hline & & Hartley & 89.70 & 90.00 & 0.9928 & 0.9950 & 39.9008 & 2.0386 \\
\hline & & Fusiello & 90.00 & 89.81 & 1.0000 & 0.9951 & 0.3315 & 0.2486 \\
\hline & & Mallon & $\mathrm{n} / \mathrm{a}$ & $\mathrm{n} / \mathrm{a}$ & $\mathrm{n} / \mathrm{a}$ & $\mathrm{n} / \mathrm{a}$ & $\mathrm{n} / \mathrm{a}$ & $\mathrm{n} / \mathrm{a}$ \\
\hline
\end{tabular}

[6] J. Gluckman and S.K. Nayar. Rectifying transformations that minimize resampling effects. CVPR, 1:111, 2001.

[7] R.I. Hartley. In defense of the eight-point algorithm. IEEE TPAMI Intelligence, 19(6):, 1997.

[8] R.I. Hartley. Theory and practice of projective rectification. IJCV, 35(2):115-127, 1999.

[9] R.I. Hartley and A. Zisserman. Multiple View Geometry in Computer Vision. Cambridge University Press, ISBN: 0521540518, second edition, 2004.

[10] C. Loop and Z. Zhang. Computing rectifying homographies for stereo vision. CVPR, 1:, 1999.

[11] David G. Lowe. Distinctive image features from scale-invariant keypoints. IJCV, 60(2):, 2004.

[12] J. Mallon and Paul F. Whelan. Projective rectification from the fundamental matrix. Image and Vision Computing, 23:643-650, 2005.

[13] L. Moisan and B. Stival. A probabilistic criterion to detect rigid point matches between two images and estimate the fundamental matrix. IJCV, 57(3):201-218, 2004.

[14] Daniel Oram. Rectification for any epipolar geometry. BMVC, 2001.

[15] T. Papadopoulo and M.I.A. Lourakis. Estimating the jacobian of the singular value decomposition:theory and application. ECCV, 1:554-570, 2000.

[16] Koch R. Pollefeys M. and Van Gool L. A simple and efficient rectification method for general motion. ICCV, 1:496-501, 1999.

[17] W.H. Press, S.A. Teukolsky, W.T. Vetterling, and B.P. Flannery. Numerical Recipes. The Art of Scientific Computing. Third Edition. Cambridge University Press, 2007. 